\title{
Organogels as nutraceutical carriers: Effect of addition of lecithin, tocopherols, and EPA/DHA on properties of candelilla wax-soybean oil organogels
}

\author{
Nydia E. Buitimea-Cantúa ${ }^{1}$, Daniel Arellano², Sergio O. Serna-Saldívar ${ }^{1}$, and Génesis V. \\ Buitimea-Cantúa ${ }^{3}$ \\ ${ }^{1}$ Instituto Tecnologico y de Estudios Superiores de Monterrey \\ ${ }^{2}$ University of Campinas \\ ${ }^{3}$ Affiliation not available
}

September 25, 2021

\begin{abstract}
Organogels are semisolid and 3D systems in which both an organic liquid solvent and other dispersed particles are entrapped. Today there is not much information about the potential of organogels as carriers of lipophilic nutraceuticals. Therefore, in this work, the preparation and characterization of soybean oil-based organogels with candelilla wax (CW) and lecithin, tocopherol, or EPA/DHA were studied. Soybean oil was structured with $0.5,1.0,2.0$, and $4.0 \% \mathrm{w} / \mathrm{w}$ of CW. In the organogels, the visual appearance, thermal stability, color parameters, microstructure, hardness, and rheological properties were analyzed. Results showed that soybean oil was successfully structured into an array of solid-like organogels varying in concentrations of CW and added nutraceutical compounds. Increasing CW concentration yielded stronger 3D-network gel formation (type 5 or solid organogel). All organogels were stable at 5,25 , and $35^{\circ} \mathrm{C}$ during the cyclization process. The nutraceuticals affected the color parameters and 3D network structure. Larger crystalline spherulites and a uniform microstructure were observed, especially in organogels formulated with $4.0 \% \mathrm{w} / \mathrm{w}$ of $\mathrm{CW}$ and lecithin. In comparison with the control, organogels $(4.0 \% \mathrm{w} / \mathrm{w}) \mathrm{with}$ tocopherol or EPA/DHA required lower shear stress to flow whereas lecithin required higher shear stress to flow. Texture analysis showed that organogels with nutraceuticals presented higher hardness, this behavior was attributed to the formation of a larger and softer crystal networks. In conclusion, soybean oil-based organogels formulated with lecithin, tocopherol, or EPA/DHA yielded stable organogels with promising properties as nutraceutical carriers.
\end{abstract}

\section{Hosted file}

Organogels as nutraceutical carriers FINAL.docx available at https://authorea.com/users/ 436122/articles/538674-organogels-as-nutraceutical-carriers-effect-of-addition-oflecithin-tocopherols-and-epa-dha-on-properties-of-candelilla-wax-soybean-oil-organogels 